\title{
Evaluation of Molecular Positive Margins Using Surrogate P53 and Retinoblastoma Protein Expression and Correlation with Surgical Outcomes in Oral Squamous Cell Carcinoma
}

\author{
Bimlesh Thakur ${ }^{1}$, Ashish Singhal ${ }^{2}$, Akash Agarwal $^{3}$, Rahat Hadi ${ }^{4}$, Rajshekar Puttaswamy ${ }^{5}$, Nuzhat Hussain ${ }^{6}$ \\ 1, 2, 3, 4, 5 Department of Surgical Oncology, Dr. Ram Manohar Lohia Institute of Medical Sciences, \\ Lucknow, Uttar Pradesh, India. ${ }^{6}$ Department of Pathology, Dr. Ram Manohar Lohia Institute of \\ Medical Sciences, Lucknow, Uttar Pradesh, India.
}

\section{ABSTRACT}

\section{BACKGROUND}

p53 mutation and $\mathrm{Rb}$ over-expression have been extensively studied in oral squamous cell carcinoma (OSCC) but their clinical relevance with respect to excision margins is still controversial. The purpose of the study was to determine the expression of molecular markers (p53\& $\mathrm{Rb}$ ) for predicting early locoregional recurrence in oral cancer.

\section{METHODS}

Histopathological specimens of 93 patients of oral cavity squamous cell carcinoma were subjected to p53 mutation and Rb protein testing in tumour and at the closest negative margin on $\mathrm{H}$ \&E using immunohistochemistry. The expression of p53 and $\mathrm{Rb}$ in tumour tissue and at excision margin was correlated with clinicopathologic parameters recurrence and survival over a 2 year follow up period.

\section{RESULTS}

p53 mutation expression in tumour tissue was associated with increased recurrence $(22.5 \%$ versus $11.3 \% \mathrm{P}=0.13)$ and mortality $(17.5 \%$ versus $5.6 \% \mathrm{P}=$ 0.056). p53 expression at margins is also associated with higher recurrence and mortality. $\mathrm{Rb}$ overexpression in tumour tissue is not significantly associated with recurrence ( $15 \%$ and $16.4 \%$ ). Rb overexpression at margins had higher recurrence ( $40 \% ; \mathrm{P}=0.627$ ) and higher mortality (60\%) in comparison to $\mathrm{Rb}$ negative cases (16.4\%versus $6.8 \%$ respectively).

\section{CONCLUSIONS}

Clinical and routine histopathological assessments of margins remain the standard method of prognosticating and planning adjuvant treatment. Determination of molecular positive margins using p53 \& $\mathrm{Rb}$ in oral cancer may aid in identifying patients at high risk of development of recurrence despite negative pathological margins.

\section{KEYWORDS}

$\mathrm{Rb}$, Margin, P53, Recurrence.
Corresponding Author:

Dr. Ashish Singhal,

Department of Surgical Oncology,

Dr. Ram Manohar Lohia Institute of

Medical Sciences, Lucknow,

Uttar Pradesh, India.

E-mail: ashishpgi@yahoo.co.in

DOI: $10.14260 / \mathrm{jemds} / 2021 / 738$

How to Cite This Article:

Thakur B, Singhal A, Agarwal A, et al. Evaluation of molecular positive margins using surrogate P53 and retinoblastoma protein expression and correlation with surgical outcomes in oral squamous cell carcinoma. J Evolution Med Dent Sci 2021;10(42):3639-3644, DOI: 10.14260/jemds/2021/738

Submission 21-07-2021,

Peer Review 06-10-2021,

Acceptance 13-10-2021,

Published 18-10-2021.

Copyright (C) 2021 Bimlesh Thakur et al. This is an open access article distributed under Creative Commons Attribution License [Attribution 4.0 International (CC BY 4.0)] 


\section{BACKGROUND}

Oral squamous cell carcinoma (OSCC) is the most common malignancy of the head and neck. It contributes to more than $90 \%$ of all malignant tumours of the oral cavity and is characterized by regional and distant metastasis. The key issue with surgical management of OSCC is predicting the risk of locoregional relapse which is reported to occur in up to 20 $\%$ of cases.1,2 It is well known that positive or close margins are associated with an increased incidence of recurrence. However, recurrences do occur despite clear histopathological margins.

Demonstration of novel molecular markers may help in identifying patients who are prone to increased risk of relapse despite clear margins by routine $H \& E$ staining. This may aid in modifying therapy and adding appropriate adjuvant treatment on a personalized basis which may help to improve outcomes. A significant drawback is a considerable time required to perform such an assessment, which limits its intraoperative usefulness. To emphasize, the molecular assessment of surgical margins is currently investigational.

\section{Objectives}

The purpose of the study was to assess the use of molecular markers on negative margins post wide excision that may assist in identifying patients who are more likely to develop locoregional recurrence with treatment naïve oral squamous cell cancer.

The primary objective was to evaluate the locoregional recurrence in patients expressing p53, Rb in tumour tissue and at the excision margin. The secondary objective was to assess the survival among those expressing these markers in the tumour tissue and at the margins.

\section{METHODS}

All patients with operable treatment naïve oral squamous cell carcinomas presenting in head and neck unit at a tertiary cancer centre undergoing surgery and meeting the inclusion criteria were recruited over 1 year and followed prospectively for two years to detect recurrence as primary objective and survival as a secondary objective. This prospective observational study was conducted between April 2018 and April 2021. The study was started after obtaining clearance from the institutional ethical committee. A total of 100 cases were enrolled. 7 patients were lost to follow up. The data of the remaining 93 patients are presented here. The following parameters of patients were recorded: age, sex, history of tobacco chewing, smoking, alcoholism, tumour site, type of treatment and recurrence during follow up. Wide excision specimens were tested for expression of p53 and retinoblastoma proteins in tumours and at the closest margin tissue of oral squamous cell carcinoma using immunohistochemistry for patients who had negative margins on routine $\mathrm{H}$ \& E staining. The patient was followed for 2 years for local recurrence / metastasis. The expression of p53 and $\mathrm{Rb}$ was correlated with local recurrence and survival.

\section{Inclusion Criteria}

1. Squamous cell cancer

2. Operable stage I to IVA

3. Treatment naïve

4. Margin negative on $\mathrm{H} \& \mathrm{E}$

5. ECOG $1-2$

6. Consenting to be part of the study and follow up

\section{Exclusion Criteria}

1. Non - squamous histology

2. Inoperable disease

3. Poor performance status

4. Positive margins on routine $\mathrm{H} \& \mathrm{E}$

\section{Experimental Technique}

Paraffin-embedded tissues were sectioned, $4 \mu \mathrm{m}$, using a microtome (Leica, Germany), and transferred to tissue bondcoated slides (Biocare, USA). After overnight incubation in a $60^{\circ} \mathrm{C}$ dry oven, paraffin-embedded sections were deparaffinised in xylene and rehydrated through graded ethanol series $100 \%, 70 \%$ and $50 \%$. Endogenous peroxidase activity was blocked with $3 \%$ hydrogen peroxide in methanol for $30 \mathrm{~min}$. Antigen retrieval was done by placing the slides in Tris - EDTA buffer ( $\mathrm{pH}$ 9.0). These sections after cooling to room temperature were incubated with p53 and retinoblastoma primary antibody at room temperature for one hour, followed by treatment with polymer-based secondary antibody kit with diaminobenzidine (DAKO, Denmark). Positive reactions were visualized using diaminobenzidine, DAB (1:50). Sections were finally counterstained with $0.1 \%$ Haematoxylin. The positive cells expressing the p53 and retinoblastoma were assessed for cytoplasmic as well as nuclear staining at lower and higher magnification (figure 1). Expression of p53 and retinoblastoma in negative histological margins was determined. Nuclear staining for p53 was both quantitatively and qualitatively evaluated. The intensity of staining was grouped into no staining $(0)$, weak $(1+)$, intermediate $(2+)$, strong $(3+)$ while the percentage of positively stained cells was calculated as a continuous variable. Intermediate to strong staining in more than $30 \%$ cancer cells was taken as mutant type expression while weak to intermediate staining in less than $30 \%$ tumour cells was interpreted wild type expression. No staining in cancer cells was taken as negative. The grade of any intensity and percentage staining for $\mathrm{Rb}$ was taken as positive.

\section{Follow Up and Data Collection}

Patients were followed for a period of 2 years for every 3 months and evaluated for recurrence or metastasis by examination which was confirmed by radiological or pathological investigations as per the merit of the case. Their clinical and pathological data were recorded in an MS excel sheet and updated periodically.

\section{Statistical Analysis}

The association between variables was assessed by chisquare test, with continuity correction for all $2 \times 2$ tables after pooling of data and by Fischer exact test for all $2 \times 2$ tables 
where P-value of chi-square test was not valid due to small counts despite pooling of data. In the presence of a small count in tables with more than two rows and / or columns, adjacent row / column data were pooled and the chi-square test was reapplied. Relative risk estimate was calculated in presence of various dichotomous variables. Comparison of quantitative data was done by using unpaired t-test if the data passed normality test or by Mann Whitney test if the data failed normality test. Statistical software SPSS 27.0.1.0 was used for statistical analysis.

\section{RESULTS}

Demographic and preoperative clinical factors like age, sex, smoking, alcohol, and tobacco habits were compared using standardized statistical tests and were found to be comparable in the present study (Table 1). An overall recurrence rate of $16.12 \%$ (15 / 93) and a 2 - year mortality of $10.7 \%$ (10 / 93) were observed. With respect to molecular margins, $43 \%$ (40 patients) had p53 expression and $21.5 \%$ (20 patients) had $\mathrm{Rb}$ protein overexpression, out of which $22.5 \%$ (9 patients) were positive for p53 at margins and 50.0 $\%$ (10 patients) were positive for Rb at margins. $22.5 \%$ (9) cases with p53 mutation developed recurrence while only $11.3 \%$ (6) cases with wild type p53 had recurrence (Table 2). Recurrence was even more profound in those with mutant p53 at margins (33.33\%; 3 cases; $\mathrm{P}=0.130$ ). However, $\mathrm{Rb}$ overexpressing tumours had almost similar recurrence rates compared to normal $\mathrm{Rb}$ protein tumours, $(20 \%, 4$ cases and $15.06 \%, 11$ cases) although those with $\mathrm{Rb}$ overexpression at margins had a higher chance of recurrence (30\%; 3 cases; $\mathrm{P}=$ 0.627). Similarly, mutant $p 53$ expressing tumours had higher mortality (17.5\%; 7 cases) than those with wild type p53 (5.6 $\%$; 3 cases) (Table 3). Those with mutant p53 at margins had a further rise in mortality (44.44\%; $4 ; \mathrm{P}=0.007) . \mathrm{Rb}$ overexpression in tumours was also reported to have higher mortality ( $25 \%$; 5 cases) with margin positive cases showing the highest rate $(60 \% ; 3$ cases $\mathrm{P}=0.035)$. However, $\mathrm{Rb}$ negative cases had significantly lower mortality rates $(6.8 \%$; 5 cases). Mean survival was significantly higher in those with wild type p53 expression (641.25 days in wild type, 498.63 days in mutant type; $\mathrm{P}=0.035$, hazard ratio $=0.029$ ). $\mathrm{Rb}$ overexpression showed a similar but statistically insignificant decreased survival. Mean recurrence-free survival also showed decreasing trend with mutant p53 and $\mathrm{Rb}$ overexpression but was not statistically significant (figure 4). Recurrence was the major cause of mortality (7 cases). 1 patient succumbed to postoperative myocardial infarction while 2 cases experienced non-institutional natural death. $46.23 \%$ of the patients received adjuvant radiotherapy or chemoradiotherapy, which was decided by routine pathological parameters. Our study found a higher p53 and $\mathrm{Rb}$ overexpression rate with increasing $\mathrm{N}$ stage but could not reach statistical significance. $\mathrm{T}$ stage and extranodal extension (ENE) were not different among different groups and were not found to be associated with either p53 mutant expression or $\mathrm{Rb}$ overexpression. The higher grade was found to have higher rates of P53 and Rb positivity but was not statistically significant. Lymphovascular emboli were associated with higher rates of mutant p53 expression and $\mathrm{Rb}$ overexpression. Perineural invasion was associated with higher rates of mutant p53 expression but not $\mathrm{Rb}$ overexpression. Other pathological factors like nuclear pleomorphism, mitotic rate, necrosis, tumour infiltrating lymphocytes, desmoplasia, tumour budding and pattern of invasion were not found to be different between the different groups. $82.79 \%$ of patients had an infiltrative pattern of invasion while pushing types were found in the rest (Table4). Regarding the tumour site, there was no significant difference between the different groups. An overall recurrence rate of $16.16 \%$ was observed, more in males $(18.42 \%$ in males and $5.8 \%$ in females). Clear margins were found in $88.17 \%$ of the patients while $11.82 \%$ had close margins. Interestingly, all the recurrences were noted in those with clear margins, though it could not reach statistical significance. This reinforces the importance of the presence of premalignant genetic alterations in surrounding histological normal mucosa. Most of the recurrences were reported in gingivobuccal complex with no statistically significant difference compared to other sites. There was an increase in recurrence rates in higher $\mathrm{T}$ and $\mathrm{N}$ stages but could not reach statistical significance.

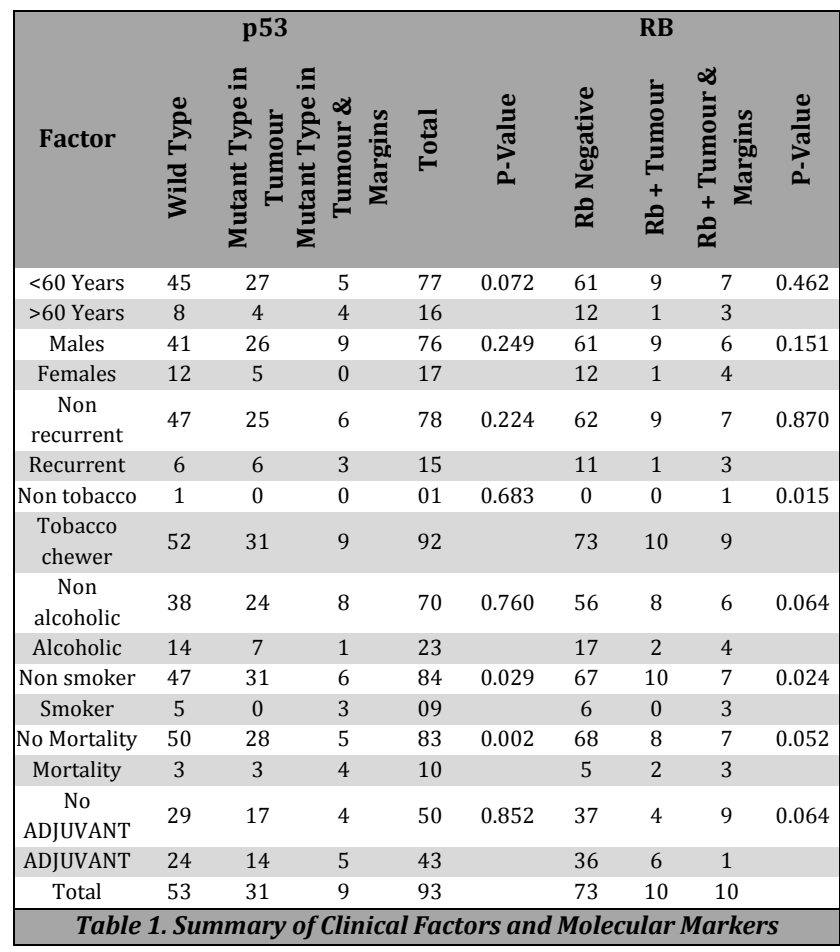

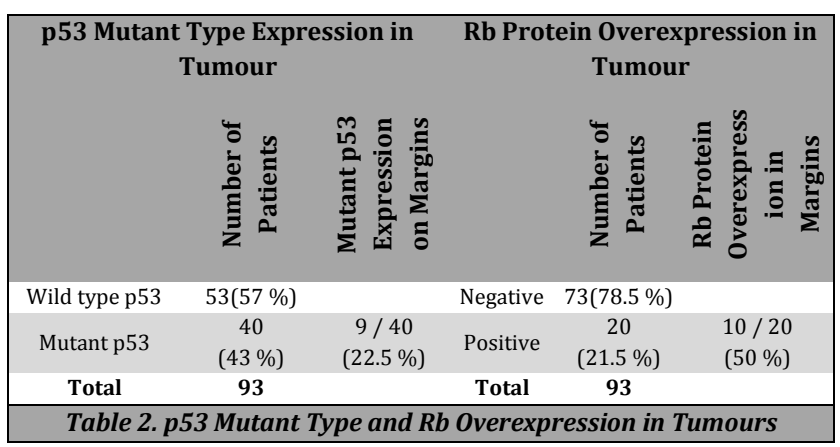




\begin{tabular}{|c|c|c|c|c|c|c|c|c|c|c|c|c|}
\hline \multicolumn{6}{|c|}{ Mutant p53 Expression and Recurrence } & \multirow{3}{*}{$\begin{array}{l}\text { Relative Risk of } \\
\text { Margin Positivity } \\
\text { (P-Value) }\end{array}$} & \multicolumn{6}{|c|}{ Rb Protein Overexpression and Recurrence } \\
\hline & \multicolumn{2}{|c|}{ p53 } & \multirow{2}{*}{ 륭 } & \multirow{2}{*}{$\begin{array}{c}P \\
\text { Value }\end{array}$} & \multirow{2}{*}{$\begin{array}{c}\text { Mutant } \\
\text { Type p53 } \\
\text { at } \\
\text { Margins }\end{array}$} & & \multicolumn{2}{|c|}{$\mathbf{R b}$} & \multirow{2}{*}{ Total } & \multirow{2}{*}{$\begin{array}{c}\mathbf{P} \\
\text { Value }\end{array}$} & \multirow{2}{*}{$\begin{array}{c}\text { Rb Over } \\
\text { expression } \\
\text { at Margins }\end{array}$} & \multirow{2}{*}{$\begin{array}{l}\text { Relative Risk of } \\
\text { Margin Positivity } \\
\text { (P-Value) }\end{array}$} \\
\hline & $\begin{array}{l}\text { Wild } \\
\text { Type }\end{array}$ & $\begin{array}{l}\text { Mutant } \\
\text { Type }\end{array}$ & & & & & Negative & Positive & & & & \\
\hline No recurrence & 47 & 31 & 78 & 0.130 & 6 & $2.3(0.224)$ & 62 & 16 & 78 & 0.627 & 7 & $2.3(0.870)$ \\
\hline \multirow{2}{*}{ Recurrence } & 6 & 9 & 15 & & 3 & & 11 & 4 & 15 & & 3 & \\
\hline & $(11.3 \%)$ & $(22.5 \%)$ & $(16.12 \%)$ & & $(33.33 \%)$ & & $(15.06 \%)$ & $(20 \%)$ & $(16.12 \%)$ & & $(30 \%)$ & \\
\hline Total & 53 & 40 & 93 & & 9 & & 73 & 20 & 93 & & 10 & \\
\hline \multicolumn{6}{|c|}{ Mutant p53 expression and mortality } & \multicolumn{7}{|c|}{ Rb protein overexpression and mortality } \\
\hline Alive & 50 & 33 & 83 & 0.007 & 5 & $6.2(0.002)$ & 68 & 15 & 83 & 0.035 & 4 & $3.5(0.052)$ \\
\hline Deaths & 3 & 7 & 10 & & 40 & & 5 & 5 & 10 & & 6 & \\
\hline Total & $\begin{array}{c}(5.6 \%) \\
53\end{array}$ & $\begin{array}{c}(17.5 \%) \\
\mathbf{4 0}\end{array}$ & $\begin{array}{c}(10.75 \%) \\
\mathbf{9 3}\end{array}$ & & $\begin{array}{c}4(4.44 \%) \\
\mathbf{9}\end{array}$ & & $\begin{array}{c}(6.8 \%) \\
73\end{array}$ & $\begin{array}{c}(25 \%) \\
20\end{array}$ & $\begin{array}{c}(10.75 \%) \\
\quad 93\end{array}$ & & $\left(\begin{array}{l}(60 \%) \\
10\end{array}\right.$ & \\
\hline
\end{tabular}

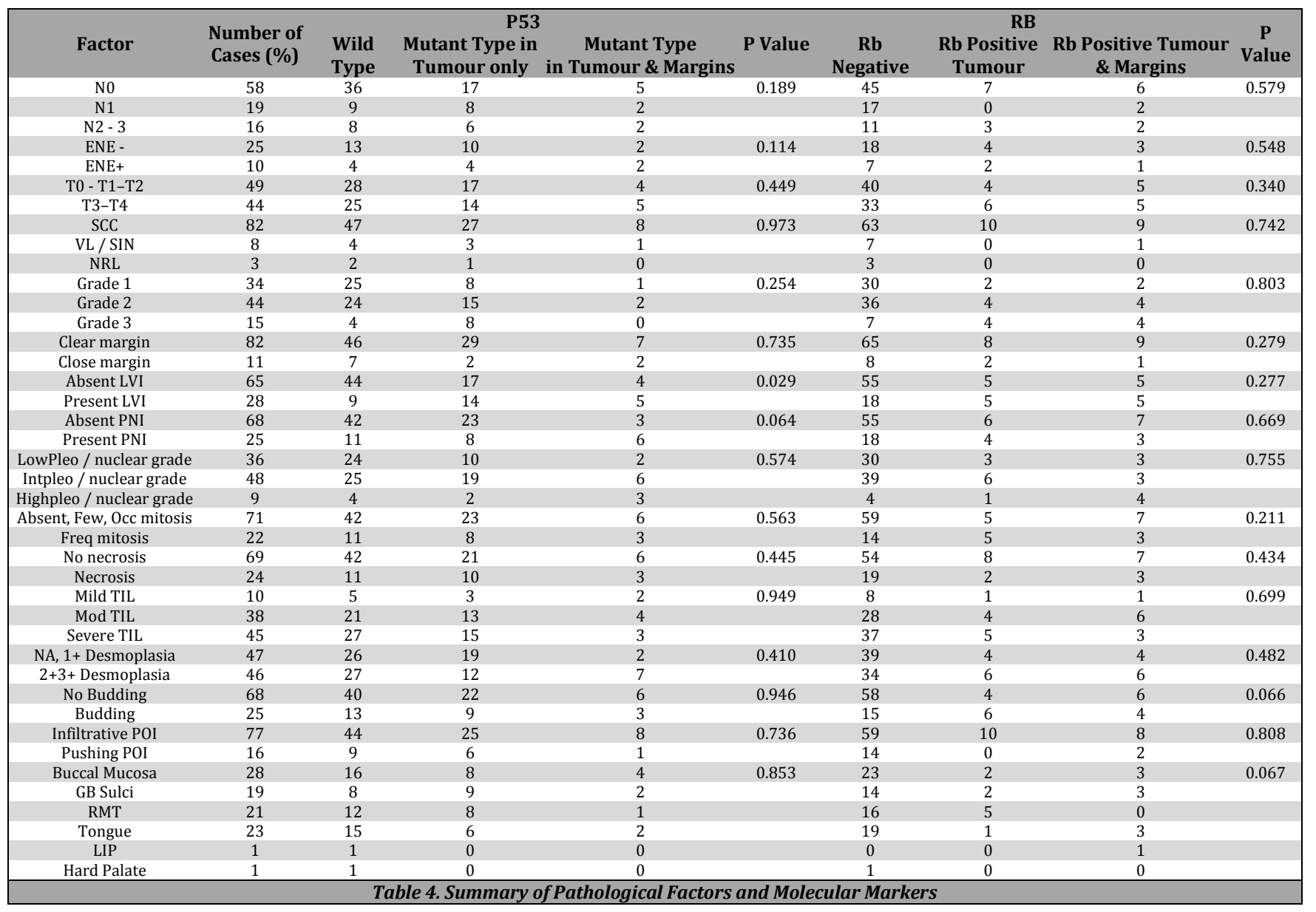

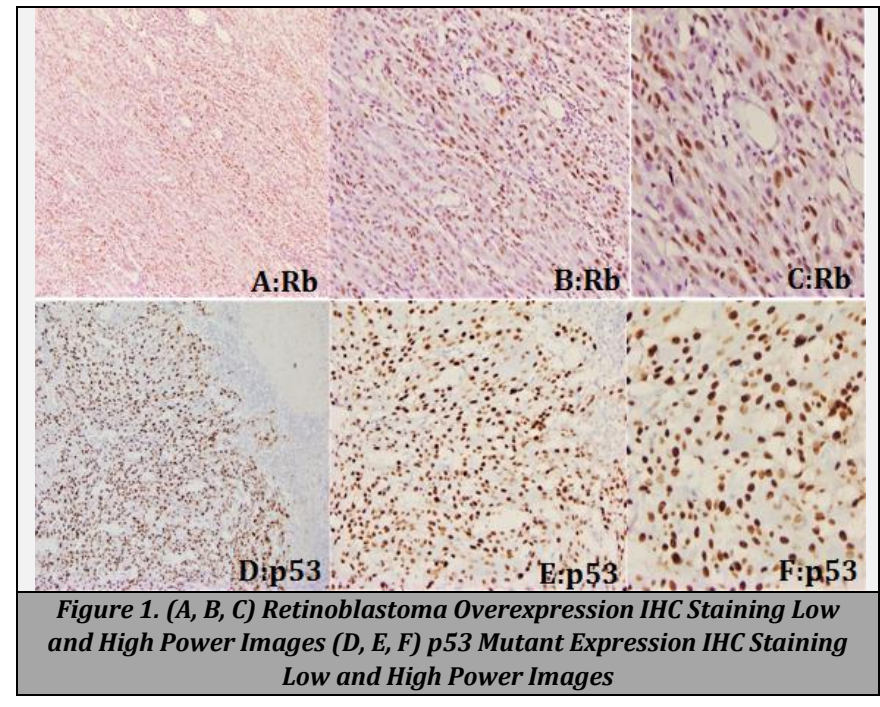

\section{DISCUSSION}

The surgical margins (SMs) or resection margins are boundaries of resection specimen excised by the surgeon to obtain local control and increase survival. The status of these resected SMs is an important and valuable tool to predict the treatment outcome. Apart from routine histopathology, the molecular assessment of resected margins referred to as molecular margins have recently gained value as tumours with histologically negative margins (HNMs) that are also known to demonstrate locoregional recurrence. ${ }^{3}$ Histological distance of $>5 \mathrm{~mm}$ from the invasive carcinoma to SMs is labelled as clear margin taking into account shrinkage at fixation and elasticity at resection. ${ }^{3}$ Various molecular markers have been employed to detect these fields of genetically altered cells.1,4,5 These fields serve as fertile 
grounds for the evolution of potentially malignant lesions as well as invasive cancer.6,7 Majority of these altered fields can be identified through immunohistochemistry (IHC) or genetic analysis. ${ }^{7}$ Determining the "molecular status" of the SMs is one of the newer diagnostic methods employed in OSCC and studies have shown that subjecting the SMs for molecular analysis helps to determine the adequacy of tumour tissue removal. Overexpression of tumour suppression genes (such as p53), oncogenes (such as epidermal growth factor receptor), and proto-oncogenes (like Her - 2) in margins reported to be cancer-free on routine histopathological examination explains the initiation of premalignant and malignant changes at these margins, which may further result in recurrence and second primary tumours. $8,9,10,11$ In a study by Von Houten et al. the absence of TP53 - mutated DNA in surgical margins was significantly associated with higher local and locoregional recurrence-free survival $(\mathrm{P}=0.027$ and $\mathrm{P}=0.028$, respectively). ${ }^{12}$ Moreover, about $20 \%$ of the cases have p53 mutated DNA in the surgical margins related to the primary tumour and the presence of TP53 - mutated DNA in the surgical margins serve as an independent prognosticator for locoregional recurrence (relative risk $=7.1 ; \mathrm{P}=0.021$ ). J.A. Brennan et al. identified 25 patients with primary squamouscell carcinoma of the head and neck containing a p53 mutation with complete tumour resection based on a negative histopathological assessment. ${ }^{13}$ In 13 of these 25 patients, molecular analysis was positive for a p53 mutation in at least one tumour margin. In 5 of 13 patients with positive margins by this method (38 percent), the carcinoma had recurred locally, as compared with none of 12 patients with negative margins $(P=0.02)$. Huang $X$ et al. found the probability of developing local recurrence was significantly higher for the group with p53 mutation-positive margins when compared with the group with clear margins $(\mathrm{P}=$ 0.048) and more strongly associated with p53 mutationpositive deep molecular margins than mutation-positive mucosal molecular margins or positivity at both sites $(\mathrm{P}=$ 0.009.14 In our study, several reports have suggested that $\mathrm{Rb}$ protein function was absent in malignant oral epithelium, whereas others have indicated raised $\mathrm{Rb}$ protein during oral cancer progression. ${ }^{15,16,17}$ The expression of $\mathrm{Rb}$ protein was found to increase from normal oral, precancerous lesions to cancers. A consistently high expression of $\mathrm{Rb}$ protein was seen in oral cancers, with an increase in well-differentiated and moderately differentiated tumours. Patients with combined habits of betel chewing, smoking, and alcohol consumption had a higher expression compared to those without habits. ${ }^{18,19}$ The management of tumours found to have a positive molecular margin is not clear. Radiation to the primary site did not prevent the development of local recurrence when the residual tumour harboured a p53 gene mutation. ${ }^{20}$ Surgical resection is impractical as the size of resection is large and ill-defined. Intense surveillance and individual case base modification in treatment protocol remain the best available option. The presence of genetic alterations in HNMs demands the refinement of the definition of tumour-free margins in OSCC and it is recommended to include molecular status along with histology. This would influence the therapeutic approach and predict local recurrence and survival rate.

\section{CONCLUSIONS}

Clinical and routine histopathological assessment of margins remains the standard method of prognosticating. Determination of molecular positive margins in oral cancer can aid in identifying patients at higher risk of development of recurrence. p53 and $\mathrm{Rb}$ expressions in histologically negative margins were associated with increased locoregional recurrence and mortality. Higher $\mathrm{N}$ stage, tumour grade but not $\mathrm{T}$ stage was associated with mutant type p53 expression and $\mathrm{Rb}$ protein overexpression in histologically negative margins. Further, large scale studies with longer follow up are needed to comment on the effect on overall and disease-free survival. Determination of molecular marker positivity can assist in determining the high risk of development of locoregional recurrences and mortality in selected cases.

\section{Limitations}

Even though many more molecular markers which have proven to be more accurate to predict the margin status have been implicated, our study only includes two markers. Presently the value of these markers in routine resection specimens of oral cancers is not well established yet. Nonetheless beholding the role of these genetic and molecular alterations to predict recurrence and survival, further studies, preferably well designed randomized trials are recommended that focus on validation and assessment of the clinical utility of these molecular markers.

Data sharing statement provided by the authors is available with the full text of this article at jemds.com.

Financial or other competing interests: None.

Disclosure forms provided by the authors are available with the full text of this article at jemds.com.

\section{REFERENCES}

[1] Braakhuis BJM, Bloemena E, Leemans CR, et al. Molecular analysis of surgical margins in head and neck cancer: more than a marginal issue. Oral Oncol 2010;46(7):48591.

[2] Eckardt A, Barth EL, Kokemueller H, et al. Recurrent carcinoma of the head and neck: treatment strategies and survival analysis in a $20 \mathrm{yr}$ period. Oral Oncol 2004;40(4):427-32.

[3] Kamat M, Rai BD, Puranik RS, et al. A comprehensive review of surgical margin in oral squamous cell carcinoma highlighting the significance of tumor-free surgical margins. J Cancer Res Ther 2019;15(3):449-54.

[4] Jadhav KB, Gupta N. Clinicopathological prognostic implicators of oral squamous cell carcinoma: need to understand and revise. N Am J Med Sci 2013;5(12):6719.

[5] Reis PP, Waldron L, Perez-Ordonez B, et al. A gene signature in histologically normal surgical margins is predictive of oral carcinoma recurrence. BMC Cancer 2011;11:437. 
[6] Farah CM, John $\mathrm{K}, \mathrm{Wu}$ J. Contemporary assessment and management of head and neck cancer surgical margins. In: Marcu LG, ed. Contemporary issues in head and neck cancer management. London, SE19SG United Kingdom: Intech 2015:75 - 130

[7] van Houten VMM, Leemans CR, Kummer JA, et al. Molecular diagnosis of surgical margins and local recurrence in head and neck cancer patients: a prospective study. Clin Cancer Res 2004;10(11):361420.

[8] Bilde A, von Buchwald C, Dabelsteen E, et al. Molecular markers in the surgical margin of oral carcinomas. J Oral Pathol Med 2009;38(1):72-8.

[9] Singh J, Jayaraj R, Baxi S, et al. Immunohistochemical expression levels of p53 and eIF4E markers in histologically negative surgical margins, and their association with the clinical outcome of patients with head and neck squamous cell carcinoma. Mol Clin Oncol 2016;4(2):166-72.

[10] Wang X, Chen S, Chen X, et al. Tumor-related markers in histologically normal margins correlate with locally recurrent oral squamous cell carcinoma: a retrospective study. J Oral Pathol Med 2016;45(2):83-8.

[11] Jelovac DB, Tepavčević $Z$, Nikolić $N$, et al. The amplification of c-erb-B2 in cancer-free surgical margins is a predictor of poor outcome in oral squamous cell carcinoma. Int J Oral Maxillofac Surg 2016;45(6):700-5.

[12] Van Houten VMM, Tabor MP, van den Brekel MWM, et al. Mutated p53 as a molecular marker for the diagnosis of head and neck cancer. J Pathol 2002;198(4):476-86.
[13] Brennan JA, Mao L, Hruban RH, et al. Molecular assessment of histopathological staging in squamous-cell carcinoma of the head and neck. $\mathrm{N}$ Engl J Med 1995;332(7):429-35.

[14] Huang X, Pateromichelakis S, Hills A, et al. P53 mutations in deep tissues are more strongly associated with recurrence than mutation-positive mucosal margins. Clin Cancer Res 2007;13(20):6099-106.

[15] Todd R, Hinds PW, Munger K, et al. Cell cycle dysregulation in oral cancer. Crit Rev Oral Biol Med 2002;13(1):51-61.

[16] Schoelch ML, Regezi JA, Dekker NP, et al. Cell cycle proteins and the development of oral squamous cell carcinoma. Oral Oncol 1999;35(3):333-42.

[17] Regezi JA, Dekker NP, McMillan A, et al. p53, p21, Rb, and MDM2 proteins in tongue carcinoma from patients $<35$ versus > 75 years. Oral Oncol 1999;35(4):379-83.

[18] Thomas S, Balan A, Balaram P. The expression of retinoblastoma tumour suppressor protein in oral cancers and precancers: a clinicopathological study. Dent Res J (Isfahan) 2015;12(4):307-14.

[19] Pande P, Mathur M, Shukla NK, et al. pRb and p16 protein alterations in human oral tumorigenesis. Oral Oncol 1998;34(5):396-403.

[20] Partridge M, Li SR, Pateromichelakis S, et al. Detection of minimal residual cancer to investigate why oral tumors recur despite seemingly adequate treatment. Clin Cancer Res 2000;6(7):2718-25. 